\title{
Development of Micro-Photoelectron Diffraction at SPring-8 BL25SU
}

\author{
Sugita Kenji, Naoyuki Maejima, ${ }^{*}$ and Hiroyuki Nishikawa \\ Nara Institute of Science and Technology, Takayama 8916-5, Ikoma, Nara 630-0192, Japan \\ Tomohiro Matsushita \\ JASRI/SPring-8, Koto 1-1-1, Sayo, Hyogo 679-5198, Japan \\ Fumihiko Matsui ${ }^{\dagger}$ \\ Nara Institute of Science and Technology, Takayama 8916-5, Ikoma, Nara 630-0192, Japan.
}

(Received 15 January 2016; Accepted 6 February 2016; Published 5 March 2016)

\begin{abstract}
Photoelectron diffraction is an effective method to analyze element-specific atomic structures of surfaces. Recently, the Two-dimensional photoelectron spectroscopy (2D-PES) end station at the soft x-ray beamline BL25SU, SPring- 8 was upgraded. Few-10- $\mu \mathrm{m}$ diameter focused beam is now available for measuring poly-crystalline and small crystalline samples. We show a series of photoelectron diffraction patterns from gold poly-crystalline surfaces mapped in two dimensions at an interval of $20 \mu \mathrm{m}$. The sample surface position was two-dimensionally scanned and the best crystalline quality position was searched for the measurements of full-hemisphere photoelectron diffraction patterns and spectra. [DOI: 10.1380/ejssnt.2016.59]
\end{abstract}

Keywords: Photoelectron diffraction; Soft X-ray photoelectron Spectroscopy; Gold; Polycrystalline surfaces; Scanning photoelectron diffraction microscopy

\section{INTRODUCTION}

Photoelectron diffraction is an atomic level analysis method based on the phenomenon of the quantum mechanical interference between the element specific photoelectron wave emitted from excited atom and the scattered waves by surrounding atoms $[1,2]$. The information on the local atomic structure is recorded in the photoelectron diffraction patterns. Since the escape depth of the photoelectron is limited to about few nm, we can analyze the local atomic arrangement of the crystal sample surfaces.

For the photoelectron diffraction pattern measurements, a display-type spherical mirror analyzer (DIANA) [3] with a wide acceptance solid angle of $1 \pi$ steradian $\left( \pm 60^{\circ}\right)$ shown in Fig. 1 is one of the most efficient apparatus. The obstacle ring electrodes and retarding grids work as low and high pass filters, respectively. The angular distributions of the specific kinetic energy photoelectrons and Auger electrons emitted from the sample are projected onto the fluorescent screen. By using a focused soft-x-ray beam together with a sample position scanning mechanism and DIANA, scanning microscopic photoelectron diffraction measurements from small- and/or polycrystalline samples are realized.

Here we demonstrated the two-dimensional microscopic spatial mapping of photoelectron diffraction patterns from gold poly-crystalline surfaces. This technique is now routinely used to find the best crystalline quality position on the various sample surfaces for the measurements of photoelectron diffraction patterns and spectra [4].

\footnotetext{
* Present Address: Condensed Matter Science Division, Quantum Beam Science Center, Japan Atomic Energy Agency, Koto 1-1-1, Sayo, Hyogo 679-5198, Japan

† Corresponding author: matui@ms.naist.jp
}

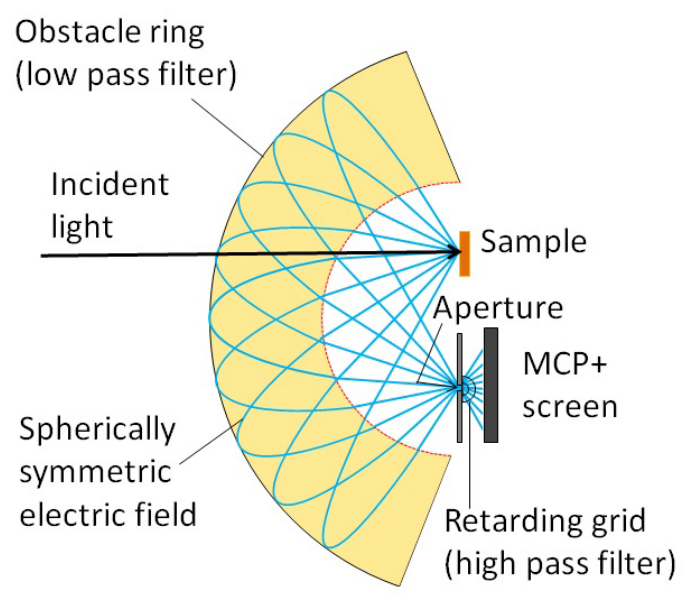

FIG. 1. Schematic diagram of a display-type spherical mirror analyzer.

\section{EXPERIMENTAL}

A gold plate was purchased from the Nilaco Corporation (purity 99.95\%, $4 \mathrm{~mm} \times 4 \mathrm{~mm}$ ). Gold sample surface was cleaned in an acetone ultrasonic bath and dried. No further treatment was done after installing into the ultrahigh-vacuum analysis chamber.

$\mathrm{Au} 4 f$ photoelectron intensity angular distributions from the gold poly-crystalline surfaces were measured using DIANA [3,5] at the circularly-polarized soft x-ray beamline BL25SU of SPring-8, Japan [6]. Details of photoelectron intensity angular distribution data processing are described elsewhere [7]. The acceptance solid angle of the analyzer was $1 \pi$ steradian $\left( \pm 60^{\circ}\right)$. Electrons emitted from the sample were energy-analyzed, and their angular distributions were projected onto the fluorescent screen. The focusing mirror dedicated to the Two-dimensional photoelectron spectroscopy (2D-PES) end station was newly installed. The size of the focused soft x-ray beam was $18 \mu \mathrm{m}$ in vertical direction and $30 \mu \mathrm{m}$ in horizontal di- 

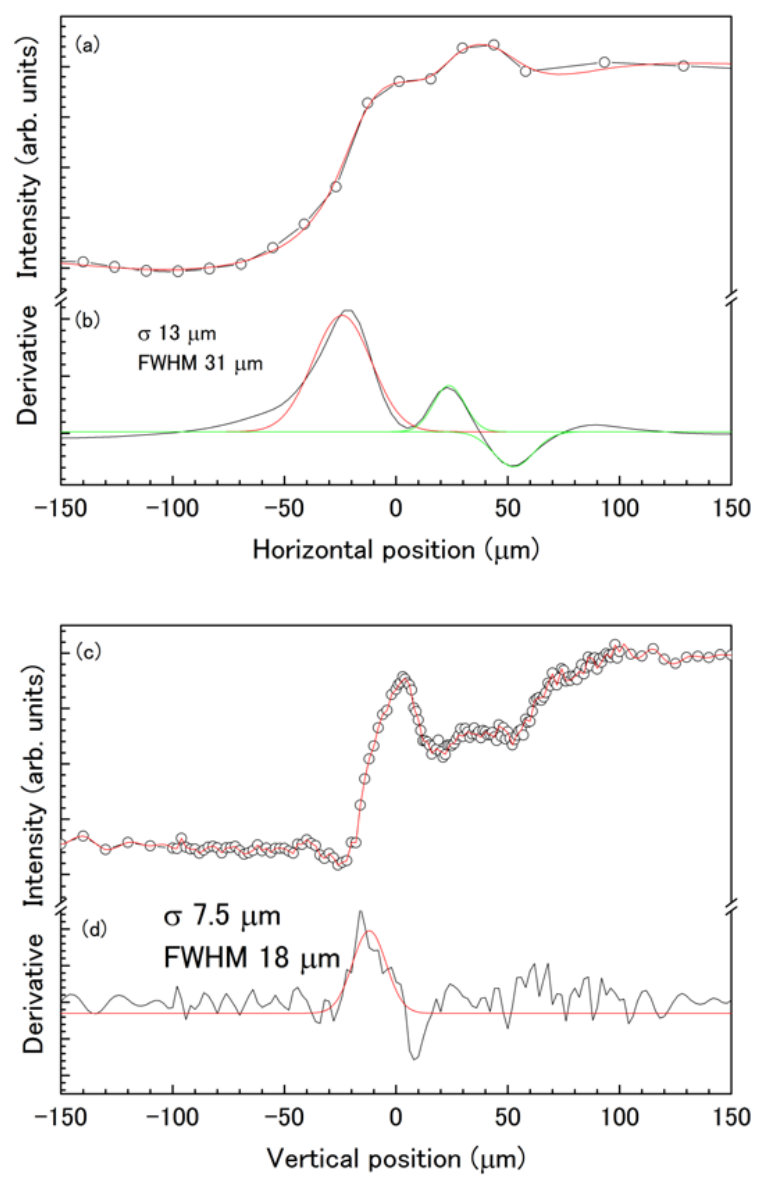

FIG. 2. X-ray beam profile characterized from the photoelectron intensity as function of beam position at the edge of $\mathrm{Si}$ wafer. (a) Photoelectron intensity variation sample position scanning along the horizontal direction. (b) Differential profile of (a). (c) and (d) Same as (a) and (b), respectively, but for the vertical direction.

rection as indicated in Fig. 2. We used the Si(111) wafer as a "knife edge". The photoelectron intensity was monitored for the evaluation of beam intensity. The light was incident from the surface normal direction. Therefore the side $\{111\}$ facet was also irradiated and as a result rich structure appeared as shown in Fig. 2(c). High photoelectron intensity was obtained when the beam irradiated the edge at the front and side surfaces. The photon energy was set to $900 \mathrm{eV}$. The photon energy resolution was about $100 \mathrm{meV}$. The electron energy resolution was determined by the analyzer energy window width. For the two-dimensional microscopic spatial mapping of photoelectron diffraction, the energy window width was set to $5 \%$ of the photoelectron kinetic energy.

\section{RESULTS AND DISCUSSIONS}

Figure 3(a) shows the summary of two-dimensional microscopic spatial mapping of Au $4 f$ photoelectron diffraction patterns from the gold surface. The size of the mapping region was $500 \mu \mathrm{m}$ in horizontal and $250 \mu \mathrm{m}$ in vertical directions. The spacing between each sampling point (a)

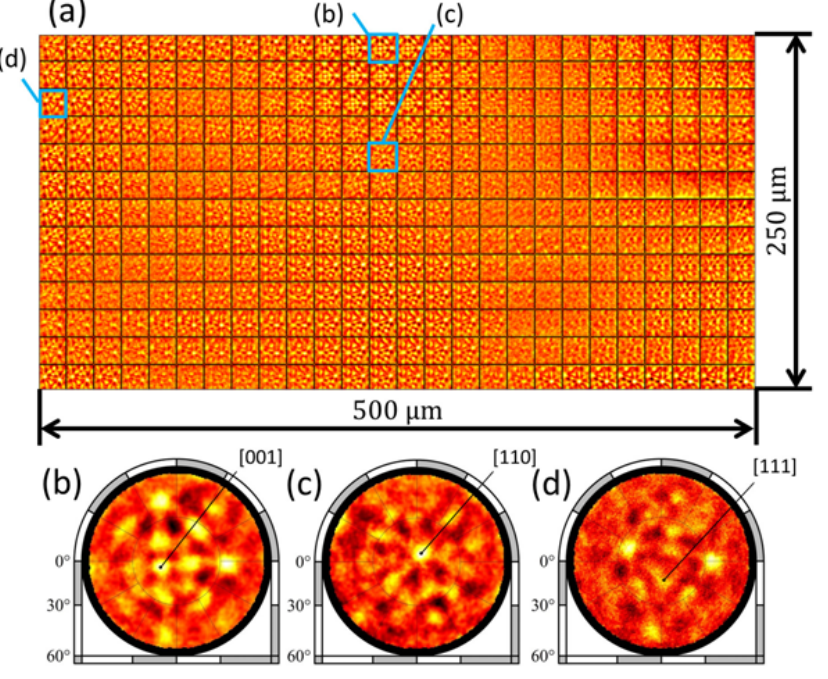

FIG. 3. (a)Two-dimensional mapping of Au $4 f$ photoelectron diffraction patterns from the gold poly-crystalline surface. Photon energy and photoelectron kinetic energy was $900 \mathrm{eV}$ and $810 \mathrm{eV}$, respectively. (b)-(d) Typical photoelectron diffraction patterns displayed in stereo projection. Corresponding patterns in the mapping (a) are marked by the blue open squares.

for the photoelectron diffraction pattern was $20 \mu \mathrm{m}$. Photon and photoelectron kinetic energies were fixed to 900 $\mathrm{eV}$ and $810 \mathrm{eV}$, respectively. The acquisition time for one photoelectron diffraction pattern was $60 \mathrm{sec}$. Total time for 338 photoelectron diffraction pattern acquisition including sample two-dimensional scanning was about 8 hours.

In this mapping, many high symmetric photoelectron diffraction patterns were observed. Three photoelectron diffraction patterns are shown in Fig. 3(b), (c), and (d) as the typical examples. They are displayed in stereo projection. Their corresponding positions are indicated in Fig. 3(a) by the open squares. Several high symmetric axes and planes were observed in $1 \pi$ steradian photoelectron diffraction patterns acquired in one single shot without rotating the sample orientation. This is important in order to efficiently map the photoelectron diffraction patterns in two dimensions as in the present measurement. It is extremely difficult to obtain similar data by the combination of a conventional electron spectrometer and a sample goniostage. It is difficult and/or time consuming to manipulate the sample during the orientation rotation keeping the irradiated position at analyzer focus point for every sampling point. Moreover, in the case of a conventional electron spectrometer, the sample azimuth and polar angles have to be scanned to obtain a wide solid angle photoelectron diffraction pattern. If the solid angle range of $60^{\circ}$ (polar) by $360^{\circ}$ (azimuth) was measured with the interval of $1^{\circ}$ step and 1 second for each point, the total acquisition time will be 6 hours for just one sampling point.

Figure 4 shows the comparison between experimental data and simulated photoelectron diffraction patterns. The symmetric operation around [001] axis was applied to the data shown in Fig. 3(b) and obtained a photoelec- 

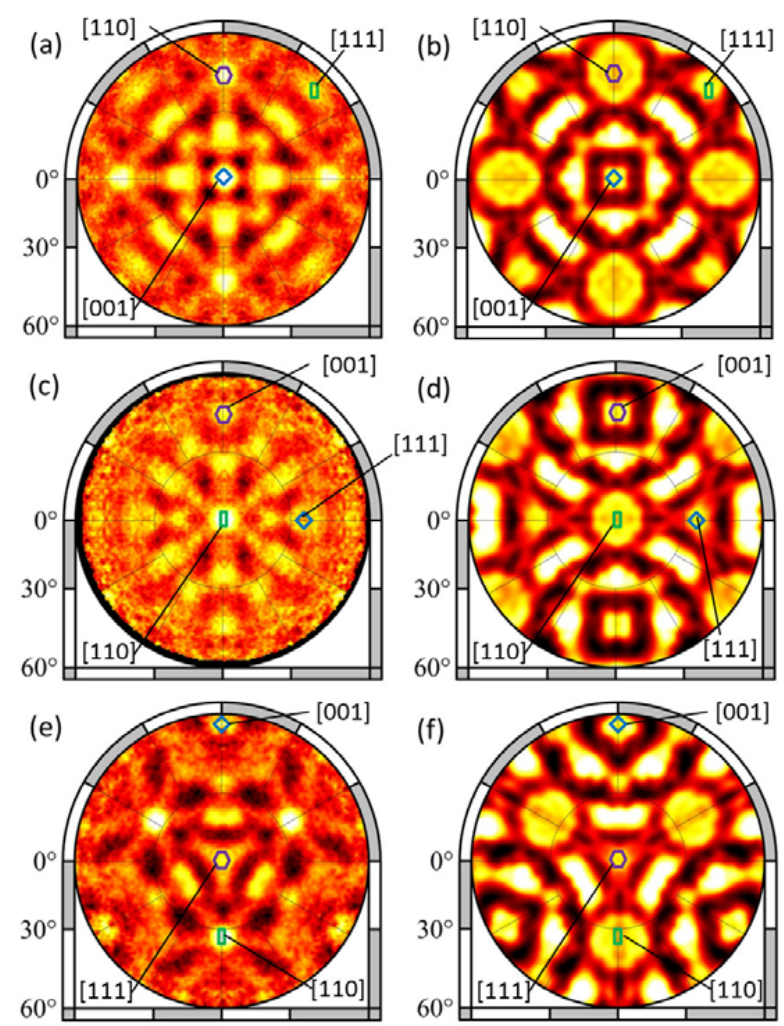

FIG. 4. (a) The experimental photoelectron diffraction pattern shown in Fig.3(b) with the symmetric operation around the [001] axis applied and (b) corresponding simulated pattern. (c) and (d) Same as (a) and (b), respectively, but for the [110] direction shown in Fig.3(c). (e) and (f) for the [111] direction shown in Fig.3(d).

tron diffraction pattern as shown in Fig. 4(a). Similarly, Fig. 4(c) and (e) are obtained by applying the symmetric operation around the [110] and [111] axes to the data shown in Fig. 3(c) and (d), respectively. Figure 4(b), (d), and (f) are the simulated photoelectron diffraction patterns by the multiple scattering pattern simulation code TMSP $[8,9]$ corresponding to Fig. 4(a), (c), and (e), respectively. Spherical 177-Au-atom cluster was used for the photoelectron diffraction simulation. The directions and the positions of forward focusing peaks as well as detailed diffraction patterns matched very well. By using such characteristic diffraction patterns, the crystal orientation for each measured point can be determined quantitatively.

Next, crystal orientation at each surveyed point was analyzed and determined. Three-dimensional crystal orientations are indicated by cubes as shown in Fig. 5. The color of the cube indicates the Miller index of the surface. The gray box indicates the position where the crystal orientation could not be determined from the photoelectron pattern probably due to the overlap of photoelectron diffractions from several different domains at the domain boundary. As shown in Fig. 5, seven crystal domains were found within the region of $0.50 \times 0.25 \mathrm{~mm}^{2}$. The distribution of the areal size of the domains in the twodimensional mapping $\bar{a}$ and the three-dimensional size (diameter) of the domains in the poly crystal $r_{3 \mathrm{D}}$ are differ-

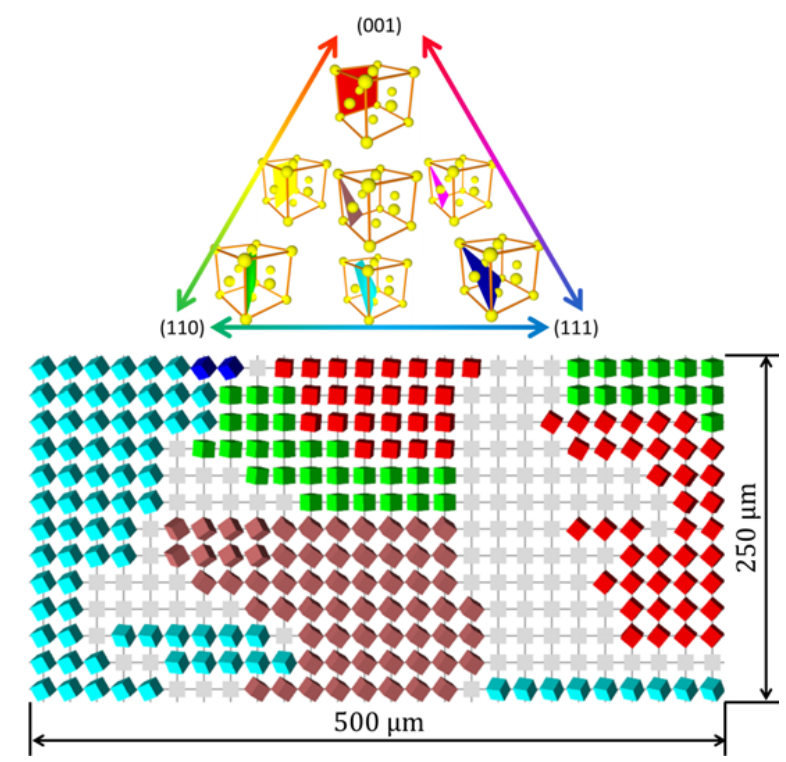

FIG. 5. Crystal orientations of the various points on the gold poly-crystalline surface determined from the two-dimensional spatial mapping of photoelectron diffraction patterns.

ent. The diameter can be estimated by using the relation $r_{3 \mathrm{D}}=\sqrt{4 \bar{a} / \pi}$. Roughly, the average cross sectional size $\bar{a}$ of a crystal domain was $1 / 56 \mathrm{~mm}^{2}$. The diameter of the crystal $r_{3 \mathrm{D}}$ was $150 \mu \mathrm{m}$. Since the number of observed crystal domains is small, it is difficult to estimate the precise three-dimensional crystal domain size using the Jeffries planimetric method [10] for instance. However, it is noteworthy that the gold plate contained crystal domains in the order of sub-mm scale. For evaluating the Fermi level for the photoelectron spectroscopy measurement, a focused x-ray or ultraviolet beam is not appropriate when using such a gold surface with large crystal domains.

\section{CONCLUSIONS}

In conclusion, we have measured a series of twodimensional photoelectron angular distributions from a poly-crystalline gold surface using a focused soft x-ray beam and a display-type analyzer. Micro-photoelectron diffractions were obtained and crystal orientation for each point was determined. As a result, crystal domains with diameter of sub-mm scale are found in the Au mapping data.

\section{ACKNOWLEDGMENTS}

This work was performed with the approval of the Japan Synchrotron Radiation Research Institute (Proposal No. 2014B1454 and 2015A0124). The authors deeply thank Dr. Takayuki Muro, Dr. Tetsuya Nakamura, Dr. Yoshinori Kotani, and Dr. Toyohiko Kinoshita for their support in the experiments. This research was supported by the Ministry of Education, Science, Sports and Culture, Grant-in-Aid for Scientific Research (B), 25287075, 2013 and JSPS Grant-in-Aid for Scientific Re- 
search on Innovative Areas "3D Active-Site Science": 261050072604.

[1] C. S. Fadley, J. Electron Spectrosc. Relat. Phenom. 178179, 2 (2010).

[2] D. P. Woodruff, J. Electron Spectrosc. Relat. Phenom. 178-179, 186 (2010).

[3] H. Daimon, Rev. Sci. Instrum. 59, 545 (1988).

[4] F. Matsui, M. Fujita, T. Ohta, N. Maejima, H. Matsui, H. Nishikawa, T. Matsushita, and H. Daimon, Phys. Rev. Lett. 114, 015501 (2015).

[5] M. Kotsugi, Y. Miyatake, K. Enomoto, K. Fukumoto, A. Kobayashi, T. Nakatani, Y. Saitoh, T. Matsushita, S. Imada, T. Furuhata, S. Suga, K. Soda, M. Jinno, T. Hirano, K. Hattori, and H. Daimon, Nucl. Instrum. Methods
A467-A468, 1493 (2001)

[6] Y. Saitoh, H. Kimura, Y. Suzuki, T. Nakatani, T. Matsushita, T. Muro, T. Miyahara, M. Fujisawa, K. Soda, S. Ueda, H. Harada, M. Kotsugi, A. Sekiyama, and S. Suga, Rev. Sci. Instrum. 71, 3254 (2000).

[7] F. Matsui, T. Matsushita, and H. Daimon, J. Electron Spectrosc. Relat. Phenom. 195, 347 (2014).

[8] Total-analysis Multiple Scattering Program [http://sourceforge.jp/projects/tmcoca/].

[9] T. Matsushita, F. Matsui, H. Daimon, and K. Hayashi, J. Electron Spectrosc. Relat. Phenom. 178-179, 195 (2010).

[10] Z. Jeffries, A. H. Kline, and E. B. Zimmer, Trans. AIME 54, 594 (1916). 